ORIGINAL

\title{
Low skeletal muscle mass is associated with insulin resistance, diabetes, and metabolic syndrome in the Korean population: The Korea National Health and Nutrition Examination Survey (KNHANES) 2009-2010
}

\author{
Seong-Su Moon ${ }^{1), 2)}$ \\ 1) Department of Internal Medicine, Dongguk University College of Medicine, Gyeongju, South Korea \\ ${ }^{2)}$ Medical Institute of Dongguk University, Gyeongju, South Korea
}

\begin{abstract}
Sarcopenia is an emerging risk factor for metabolic disorders. No study of the association of sarcopenia with insulin resistance, diabetes, and metabolic syndrome (MS) according to age group and obesity status in the general population has been reported. We investigated these associations in the Korean population. Participants included 4558 males and 5874 females, who were $\geq 20$ years of age or older from the fourth and fifth Korea National Health and Nutritional Examination Surveys of the Korean population (2009 and 2010). Age was categorized according to three groups (20-39, $40-59$, and $\geq 60$ years). Obesity was defined according to body mass index. Sarcopenia was defined as the appendicular skeletal muscle mass (ASM) divided by weight (Wt) $(\%)$ of $>2 \mathrm{SD}$ below the sex-specific mean for young adults. Homeostasis model assessment of insulin resistance (HOMA-IR) was calculated. After adjustment for confounding variables, sarcopenia showed a significant association with HOMA-IR in the non-obese group $(P<0.001)$. Sarcopenia was found to be a risk factor for diabetes in the non-obese group $(O R, 2.140 ; 95 \% C I, 1.549-2.956 ; P<0.001)$. Sarcopenia also showed an association with MS in the non-obese group $(O R, 2.209 ; 95 \% C I, 1.679-2.906 ; P<0.001)$, but not in the obesegroup. However, these results were not relevant to young age group. In conclusion, sarcopenia showed an association with insulin resistance, diabetes, and MS, in the non-obese population. Sarcopenia may be an early predictor for diabetes and MS susceptibility in the non-obese population, particularly in elderly people.
\end{abstract}

Keywords: Sarcopenia, Insulin resistance, Diabetes, Metabolic syndrome

SARCOPENIA specifically refers to the loss of muscle mass associated with aging [1-3]. Sarcopenia is known to be related to metabolic disorders, including obesity, insulin resistance, and diabetes [4-7]. Obesity is believed to be the most important underlying cause of insulin resistance. By turns, insulin resistance has been suggested as a pathogenetic mechanism of sarcopenia $[5,8]$.

Inversely, sarcopenia may cause an increase in insulin resistance without obesity. Because skeletal muscle is a primary site for glucose uptake and deposition [9], sarcopenia promotes insulin resistance, leading to

Submitted Jun.11, 2013; Accepted Sep. 19, 2013 as EJ13-0244 Released online in J-STAGE as advance publication Oct. 1, 2013

Correspondence to: Seong-Su Moon, M.D., Ph.D., Assistant Professor, Department of Internal Medicine, Dongguk University College of Medicine, Dongdae-ro 87, Gyeongju, Gyeongbuk Province 780-350, South Korea. E-mail: drmoonss@hanmail.net development of metabolic syndrome (MS) and diabetes [4]. In addition, myokines secreted by skeletal muscle have been suggested to prevent insulin resistance by counteracting the metabolic effect of adipokines produced in adipose tissue [10]. Paucity of myokines from sarcopenia could play a role in development of insulin resistance [11]. Improvement of whole-body lean mass in elderly subjects with sarcopenia has been reported to result in increased insulin sensitivity [12].

Insulin resistance is also an underlying mechanism for type 2 diabetes and MS, which is characterized by a constellation of cardiovascular risk factors. including obesity, hypertension, dyslipidemia, and dysglycemia [13]. Therefore, it could be inferred that sarcopenia induces type 2 diabetes or MS through increasing insulin resistance without obesity.

Sarcopenia has so far been regarded as a geriatric and aging disease; therefore, it has been studied mainly 
among members of the elderly population. Total lean body mass shows a decline after the age of 45, however, onset of the decline in total lean body mass percentage of total body mass can be detected as early as the third decade; therefore, among younger members of the population, there must be some individuals with sarcopenia [14]. However, etiology of sarcopenia could differ among age groups. Muscle type II fibers, which are less sensitive to insulin than type I fibers, have shown greater vulnerability to atrophy with aging than type I fibers $[15,16]$. In addition, the relationship of sarcopenia to insulin resistance or pre-diabetes has been suggested to differ according to age and coexistence of obesity [17].

To the best of our knowledge, no study of the association of sarcopenia with insulin resistance, diabetes, and metabolic syndrome according to age group and obesity status in the general population has been reported. In order to elucidate these relationships, we investigated the relationship of sarcopenia with insulin resistance, diabetes, and metabolic syndrome in the Korean population based on the Korea National Health and Nutrition Examination Survey conducted in 2009 and 2010.

\section{Subjects and Methods}

\section{Study population}

This study was based on data acquired during the third year (2009) of the Korea National Health and Nutrition Examination Survey (KNHANES) IV (20072009) and the first year (2010) of KNHANES V (20102012). These surveys have been conducted periodically since 1998, using a rolling sampling design involving a complex, stratified, multistage, probability-cluster survey of a representative sample of the non-institutionalized civilian population in order to assess the health and nutritional status of the Korean population [18-20]. The survey, which was conducted by the Korean Ministry of Health and Welfare, consists of the health interview survey, the health examination survey, and the nutrition survey. These surveys were completed by 10,078 (79.2\% of the total target population of 12,722) participants and $8473(77.5 \%$ of the total target population of 10.938$)$ participants in 2009 and 2010, respectively. The current cross-sectional study was restricted to participants who were $\geq 20$ years of age who completed the health examination survey, including whole body DXA $(n=10432)$. During conduct of the survey, blood samples were collected from each participant in the morning following an overnight fast and were processed, immediately refrigerated, and transported in cold storage to the Central Testing Institute in Seoul, Korea. Analysis of all blood samples was performed within $24 \mathrm{~h}$ after transportation. Measurements of the concentration of glucose, high density lipoprotein (HDL) cholesterol, low density lipoprotein (LDL) cholesterol, total cholesterol, and triglycerides were performed using a Hitachi automatic analyzer 7600 (Tokyo, Japan). Immunoradiometric assay using a 1470 WIZARD gamma-counter (PerkinElmer, Finland) was used for measurement of insulin. Homeostasis model assessment of insulin resistance (HOMA-IR) was calculated using the following formula: HOMA-IR=fasting plasma glucose $(\mathrm{mg} / \mathrm{dL}) \times$ fasting insulin $(\mu \mathrm{IU} / \mathrm{mL}) / 405$ [21]. Obesity was defined as a body mass index 27.5 $\mathrm{kg} / \mathrm{m}^{2}$, the cutoff associated with increased mortality rates in Asians, including Koreans [22, 23]. Waist circumference was measured at the mid-point between the lower margin of the last palpable rib and the top of the iliac crest at the end of a normal expiration with the arms relaxed at the sides. According to the National Cholesterol Education Program Adult Treatment Panel III criteria [24] and the Korean abdominal obesity criteria for waist circumference [25], subjects were classified as having MS if they had any three of the following five characteristics: central obesity (waist circumference $\geq 90 \mathrm{~cm}$ in men and $\geq 85 \mathrm{~cm}$ in women), hypertriglyceridemia (triglycerides $\geq 150 \mathrm{mg} / \mathrm{dL}$ or triglyceride-lowering medication), HDL cholesterol $(<40 \mathrm{mg} / \mathrm{dL}$ in males or $<50 \mathrm{mg} / \mathrm{dL}$ in females), hypertension $(\geq 130 \mathrm{mmHg}$ systolic or $\geq 85 \mathrm{mmHg}$ diastolic pressure, or use of antihypertensive medication), dysglycemia (fasting plasma glucose $\geq 100 \mathrm{mg} / \mathrm{dL}$ or use of anti-hyperglycemic medication, or previously diagnosed type 2 diabetes). The study was approved by the Institutional Review Board of the Korea Center for Disease Control and prevention (2012-01EXP-01-2C).

\section{Definition of sarcopenia}

DualenergyX-rayabsorptiometry(DXA)(DiscoveryW; Hologic, Waltham, MA,USA) was used for measurement of appendicular skeletal muscle mass (ASM) [26]. Sarcopenia was defined as ASM divided by body weight (ASM/Wt) (\%) more than two standard deviations (SD) below mean value of sex-specific normal young people $[27,28]$. The definition was modified from the studies of Janssen et al. [29]. The sex-specific young reference group included 3343 healthy adults aged 20-40 yr (1449 men, 1894 women). As cutoff points, 
the two SDs of sex-specific normal young people were $26.98 \%$ in men and $21.14 \%$ in women, respectively. All participants in this survey signed an informed consent form.

\section{Statistical analyses}

Levels of HOMA-IR were log-transformed because their distributions were positively skewed. Geometric means with standard errors of HOMA-IR were calculated using analysis of covariance (ANCOVA) after adjustment for age, sex, region, smoking, alcohol consumption, regular exercise, and family income. Age was categorized according to three groups (20-39 years, $40-59$ years, and $\geq 60$ years). Logistic regression models were used for calculation of multivariate-adjusted odds ratios (ORs). Adjusted variables included age, sex, region, smoking, alcohol consumption, regular exercise, and family income. Subgroup analyses stratified according to age, sex, region, smoking, alcohol drinking, regular exercise, and family income were performed. Region was categorized as rural and urban. Among the 16 administrative districts where this survey was conducted, Seoul, Gyeonggi, and six other metropolitan cities (Busan, Daegu, Incheon, Gwangju, Daejeon, and Ulsan) were grouped as urban areas. The remaining regions were grouped as rural areas. Smoking status was divided into three categories: never, former, and current smoker. Alcohol consumption was indicated as 'yes' for participants who consumed at least two units of alcohol every week over the last year. Regular exercise was indicated as 'yes' when the participant performed moderate or strenuous exercise on a regular basis, regardless of indoor or outdoor exercise (for more than $30 \mathrm{~min}$ at a time and more than five times per week in the case of moderate exercise, such as swimming slowly, tennis doubles, volleyball, badminton, table tennis, and carrying light objects; for more than $20 \mathrm{~min}$ at a time in the case of strenuous exercise, such as running, climbing, cycling fast, swimming fast, football, basketball, jump rope, squash, tennis singles, and carrying heavy objects or when subject walked for more than $30 \mathrm{~min}$ at a time and more than five times per week. Family income was categorized into four groups according to quartile. All tests were two-sided, and $P<0.05$ was considered statistically significant. Statistical Package for Social Science 15.0 software (SPSS, Chicago, IL, USA) was used in performance of all analyses.

\section{Results}

\section{Insulin resistance according to the category of sar- copenia and obesity}

The baseline characteristics according to sarcopenic status are shown in Table 1. Among sarcopenic participants, compared with nonsarcopenic participants, higher HOMA-IR was observed only in the non-obese group $(P<0.001)$. While sarcopenic subjects had a higher HOMA-IR than those without sarcopenia in 40-59 years and $\geq 60$ years age group of the non-obese group ( $P=0.002$ and $<0.001$, respectively), after adjustment for age, sex, region, smoking, alcohol consumption, exercise, and family income, the 20-39 years age group showed the tendency without significance $(2.20 \pm 1.07$ vs. $1.98 \pm 1.01$, among sarcopenic and nonsarcopenic subjects, respectively, $P=0.144)$. Subjects in the obese group did not show that tendency $(P=0.238)$ (Table 2$)$.

\section{Prevalence of diabetes according to the category of sarcopenia and obesity}

Prevalence of diabetes was higher among sarcopenic participants than among nonsarcopenic participants only in the non-obese group $(23.1 \% v s .7 .8 \%$, $P<0.001)$. Sarcopenic subjects had a higher prevalence of diabetes than those without sarcopenia only in $\geq 60$ years age group of the non-obese group $(O R=2.448$; $95 \% C I, 1.695-3.538 ; P<0.001)$. No significant association was observed between diabetes and sarcopenia in other age groups (Table 3 ).

\section{Prevalence of MS according to the category of sar- copenia and obesity after stratification}

Prevalence of MS was higher among sarcopenic subjects, compared to nonsarcopenic subjects in the non-obese group $(O R=2.209 ; 95 \% C I, 1.679-2.906$; $P<0.001$ ), however, that relationship was not observed in the obese group. After stratification according to age, sex, region, smoking, alcohol drinking, regular exercise, and family income, MS was found to be more prevalent among nonobese and sarcopenic participants in every subgroup except the 20-39 years age group and the 75th-100th family income group, compared with nonobese and nonsarcopenic subjects after adjustment for confounding variables (Table 4).

\section{Association of sarcopenia with individual compo- nents of MS in non-obese participants}

When we attempted to determine which individual 
Table 1 Baseline characteristics of participants according to sarcopenic status ${ }^{\mathrm{a}}$

\begin{tabular}{|c|c|c|c|}
\hline & Non-Sarcopenic & sarcopenic & $P$ \\
\hline No. of total participants & 9988 & 444 & \\
\hline Age, years & $48.3 \pm 15.5$ & $59.8 \pm 14.3$ & $<0.001$ \\
\hline Men, $\%$ & $4392(44.0)$ & $166(37.4)$ & 0.006 \\
\hline Body height, $\mathrm{cm}$ & $162.6 \pm 9.1$ & $157.4 \pm 8.8$ & $<0.001$ \\
\hline Body weight, $\mathrm{kg}$ & $62.4 \pm 11.2$ & $67.6 \pm 12.2$ & $<0.001$ \\
\hline BMI, kg/cm² & $23.5 \pm 3.2$ & $27.2 \pm 3.7$ & $<0.001$ \\
\hline $\mathrm{WC}, \mathrm{cm}$ & $80.4 \pm 9.6$ & $91.1 \pm 10.0$ & $<0.001$ \\
\hline $\mathrm{FPG}, \mathrm{mg} / \mathrm{dL}$ & $97.3 \pm 22.1$ & $106.5 \pm 26.5$ & $<0.001$ \\
\hline Insulin $\mu \mathrm{IU} / \mathrm{mL}$ & $10.0 \pm 5.2$ & $12.8 \pm 8.1$ & $<0.001$ \\
\hline HOMA-IR ${ }^{b}$ & $2.46 \pm 1.87$ & $3.42 \pm 2.46$ & $<0.001$ \\
\hline T-chol, mg/dL & $187.5 \pm 35.8$ & $199.5 \pm 40.7$ & $<0.001$ \\
\hline Triglyceride, mg/dL & $134.6 \pm 114.9$ & $154.4 \pm 98.2$ & $<0.001$ \\
\hline HDL-C, mg/dL & $52.5 \pm 12.7$ & $50.0 \pm 12.4$ & $<0.001$ \\
\hline LDL-C, mg/dL & $111.9 \pm 30.9$ & $118.5 \pm 35.4$ & 0.018 \\
\hline Metabolic syndrome & $2333(23.4)$ & $254(57.2)$ & $<0.001$ \\
\hline ASM, $\mathrm{kg}$ & $17.8 \pm 4.7$ & $15.1 \pm 4.1$ & $<0.001$ \\
\hline $\mathrm{ASM} / \mathrm{Wt}, \%$ & $28.4 \pm 4.1$ & $22.2 \pm 3.0$ & $<0.001$ \\
\hline Regions $^{\mathrm{c}}$ & & & 0.691 \\
\hline Rural area & $2450(24.5)$ & $105(23.6)$ & \\
\hline Urban area & $7538(75.5)$ & $339(76.4)$ & \\
\hline Smoking status ${ }^{\mathrm{d}}$ & & & 0.001 \\
\hline Never & $5792(58.0)$ & $272(61.3)$ & \\
\hline Former & $1060(10.6)$ & $65(14.6)$ & \\
\hline Current & $3136(31.4)$ & $107(24.1)$ & \\
\hline Alcohol consumption ${ }^{\mathrm{e}}$ & & & 0.178 \\
\hline Yes & $2186(21.9)$ & $85(19.1)$ & \\
\hline No & $7802(78.1)$ & $359(80.9)$ & \\
\hline Regular exercise $^{f}$ & & & 0.052 \\
\hline Yes & $5316(53.2)$ & $215(48.4)$ & \\
\hline No & $4672(46.8)$ & $229(51.6)$ & \\
\hline Family income ${ }^{g}$ & & & $<0.001$ \\
\hline$<25$ th & $1808(18.3)$ & $139(31.8)$ & \\
\hline 25 th to 50 th & $2411(24.5)$ & $118(27.0)$ & \\
\hline 50th to 75 th & $2824(28.6)$ & $93(21.3)$ & \\
\hline 75 th to 100 th & $2815(28.6)$ & 87 (19.9) & \\
\hline
\end{tabular}

BMI, body mass index; WC, waist circumference; FPG, fasting plasma glucose; HOMAIR, homeostasis model assessment of insulin resistance; T-chol, total cholesterol; HDL-C, high density lipoprotein cholesterol; LDL-C, low density lipoprotein cholesterol; ASM, appendicular skeletal muscle mass; ASM/Wt, appendicular skeletal muscle mass/body weight.

${ }^{a}$ Values are presented as number(\%) or mean (SD). ${ }^{b}$ Geometric mean \pm SE. ${ }^{c}$ Region was categorized as urban (Seoul, Gyeonggi, Busan, Daegu, Incheon, Gwangju, Daejeon, and Ulsan) and rural (eight other administrative districts) area. ${ }^{\mathrm{d}}$ Smoking status was divided into three categories: never, former, and current smoker. ${ }^{\mathrm{e}}$ Alcohol consumption was indicated as 'yes' for participants who consumed at least two units of alcohol every week over the last year. ${ }^{f}$ Regular exercise was indicated as 'yes' when the participant performedmoderate or strenuous exercise on a regular basis (for more than $30 \mathrm{~min}$ at a time and more than five times per week in the case of moderate exercise; for more than $20 \mathrm{~min}$ at a time in the case of strenuous exercise) or when the subject walked for more than $30 \mathrm{~min}$ at a time and more than five times per week. ${ }^{\mathrm{g}}$ Family income was categorized into four groups according to quartile. 
Table 2 Adjusted geometric means and SEs of HOMA-IR according to category of sarcopenia and obesity

\begin{tabular}{|c|c|c|c|c|c|c|}
\hline & \multicolumn{2}{|c|}{ Non-obese } & \multirow{2}{*}{$P$} & \multicolumn{2}{|c|}{ Obese } & \multirow{2}{*}{$P$} \\
\hline & Non-sarcopenic & Sarcopenic & & Non-sarcopenic & Sarcopenic & \\
\hline \multicolumn{7}{|l|}{ Total participants } \\
\hline No of participants & 8164 & 192 & & 850 & 134 & \\
\hline HOMA-IR ${ }^{\mathrm{a}}$ & $2.00 \pm 1.00$ & $2.32 \pm 1.03$ & $<0.001^{\dagger}$ & $3.02 \pm 1.02$ & $3.15 \pm 1.04$ & 0.309 \\
\hline \multicolumn{7}{|l|}{ Age group } \\
\hline \multicolumn{7}{|l|}{ 20-39 year } \\
\hline No of participants & 2942 & 28 & & 298 & 19 & \\
\hline HOMA-IR ${ }^{\mathrm{a}}$ & $1.98 \pm 1.01$ & $2.20 \pm 1.07$ & 0.144 & $3.10 \pm 1.02$ & $3.14 \pm 1.09$ & 0.892 \\
\hline \multicolumn{7}{|l|}{$40-59$ year } \\
\hline No of participants & 3256 & 70 & & 365 & 53 & \\
\hline HOMA-IR ${ }^{\mathrm{a}}$ & $1.97 \pm 1.01$ & $2.28 \pm 1.05$ & $0.002^{\dagger}$ & $2.95 \pm 1.07$ & $3.21 \pm 1.07$ & 0.219 \\
\hline \multicolumn{7}{|l|}{$\geq 60$ years } \\
\hline No of participants & 1966 & 94 & & 187 & 62 & \\
\hline HOMA-IR ${ }^{\mathrm{a}}$ & $2.10 \pm 1.01$ & $2.42 \pm 1.04$ & $<0.001^{\dagger}$ & $3.03 \pm 1.03$ & $3.03 \pm 1.06$ & 0.981 \\
\hline
\end{tabular}

Data are presented as adjusted geometric mean \pm SE.

${ }^{a}$ Adjusted for age, sex, region, smoking, alcohol consumption, exercise, and family income. ${ }^{\dagger} P<0.01$.

Table 3 Adjusted ORs and 95\% CIs of prevalence of diabetes according to category of sarcopenia and obesity

\begin{tabular}{|c|c|c|c|c|c|c|}
\hline & \multicolumn{2}{|c|}{ Non-obese } & \multirow{2}{*}{$P$} & \multicolumn{2}{|c|}{ Obese } & \multirow{2}{*}{$P$} \\
\hline & Non-sarcopenic & Sarcopenic & & Non-sarcopenic & Sarcopenic & \\
\hline \multicolumn{7}{|l|}{ Total participants } \\
\hline Case $/ \mathrm{n}$ & $704 / 8972$ & $59 / 255$ & & $154 / 1016$ & $53 / 189$ & \\
\hline Prevalence $(\%)$ & 7.8 & 23.1 & & 15.2 & 28.0 & \\
\hline Adjusted $O R(95 \% \mathrm{CI})$ & 1 & $2.140(1.549-2.956)$ & $<0.001^{\dagger}$ & 1 & $1.346(0.908-1.995)$ & 0.139 \\
\hline \multicolumn{7}{|l|}{ Age group } \\
\hline \multicolumn{7}{|l|}{ 20-39 years } \\
\hline Case/n & $24 / 3001$ & $1 / 29$ & & $14 / 303$ & $4 / 23$ & \\
\hline Prevalence $(\%)$ & 0.8 & 3.4 & & 4.4 & 17.4 & \\
\hline Adjusted $O R(95 \% \mathrm{CI})$ & 1 & $3.700(0.468-29.246)$ & 0.215 & 1 & $3.832(0.996-14.739)$ & 0.051 \\
\hline \multicolumn{7}{|l|}{$40-59$ years } \\
\hline Case $/ \mathrm{n}$ & $263 / 3560$ & $10 / 80$ & & $66 / 435$ & $12 / 66$ & \\
\hline Prevalence( $\%)$ & 7.4 & 12.5 & & 15.2 & 18.2 & \\
\hline Adjusted $O R(95 \% \mathrm{CI})$ & 1 & $1.621(0.809-3.248)$ & 0.173 & 1 & $1.223(0.604-2.479)$ & 0.576 \\
\hline \multicolumn{7}{|l|}{$\geq 60$ years } \\
\hline Case/n & $417 / 2411$ & $48 / 146$ & & $74 / 264$ & $37 / 100$ & \\
\hline Prevalence(\%) & 17.3 & 32.9 & & 28.0 & 37.0 & \\
\hline Adjusted $O R(95 \% \mathrm{CI})$ & 1 & $2.448(1.695-3.538)$ & $<0.001^{\dagger}$ & & $1.267(0.762-2.107)$ & 0.362 \\
\hline
\end{tabular}

Adjusted for age, sex, region, smoking, alcohol consumption, exercise, and family income. ${ }^{\dagger} P<0.01$.

component of MS is involved in the significant relationship between sarcopenia and MS in the non-obese group, hypertension, elevated TG, and central obesity were found to have a significant relationship with sarcopenia. In $\geq 60$ years age subgroup of the non-obese group, prevalence of hypertension, elevated TG, dysglycemia (FPG $\geq 100 \mathrm{mg} / \mathrm{dl}$ or on diabetes treatment), and central obesity was higher among sarcopenic subjects than among those without sarcopenia. In contrast, subjects in the obese group did not show that tendency (Table 5).
Insulin resistance and prevalence of diabetes and MS according to the category of sarcopenia and obesity in the elderly

To show the data of the elderly, age was categorized according to three groups (60-69, 70-79, and $\geq 80$ years). While sarcopenic subjects had higher HOMA-IR and prevalence of diabetes and MS than those without sarcopenia in the non-obese group, after adjustment for age, sex, region, smoking, alcohol consumption, exercise, and family income, subjects in the obese group did not show that tendency. Among sarcopenic partici- 
Table 4 Adjusted ORs and 95\% CIs of prevalence of MS according to category of sarcopenia and obesity after stratification by age, sex, region, smoking, alcohol consumption, exercise, or family income.

\begin{tabular}{|c|c|c|c|c|c|c|}
\hline & \multicolumn{2}{|c|}{ Non-obese } & \multirow{2}{*}{$P$} & \multicolumn{2}{|c|}{ Obese } & \multirow{2}{*}{$P$} \\
\hline & Non-sarcopenic & Sarcopenic & & Non-sarcopenic & Sarcopenic & \\
\hline Total participants & 1 & $2.209(1.679-2.906)$ & $<0.001^{\dagger}$ & 1 & $0.965(0.664-1.403)$ & 0.853 \\
\hline \multicolumn{7}{|l|}{ Age } \\
\hline 20-39 years & 1 & $2.226(0.633-7.825)$ & 0.212 & 1 & $1.055(0.435-2.562)$ & 0.905 \\
\hline $40-59$ years & 1 & $1.931(1.183-3.152)$ & $0.008^{\dagger}$ & 1 & $0.983(0.555-1.743)$ & 0.954 \\
\hline$\geq 60$ years & 1 & $2.641(1.859-3.753)$ & $<0.001^{\dagger}$ & 1 & $0.918(0.495-1.701)$ & 0.786 \\
\hline \multicolumn{7}{|l|}{ Sex } \\
\hline Men & 1 & $2.763(1.809-4.220)$ & $<0.001^{\dagger}$ & 1 & $1.564(0.793-3.038)$ & 0.197 \\
\hline Women & 1 & $1.965(1.358-2.845)$ & $<0.001^{\dagger}$ & 1 & $0.771(0.486-1.223)$ & 0.269 \\
\hline \multicolumn{7}{|l|}{ Region } \\
\hline Rural area & 1 & $2.770(1.620-4.737)$ & $<0.001^{\dagger}$ & 1 & $0.665(0.305-1.450)$ & 0.305 \\
\hline Urban area & 1 & $2.010(1.458-2.772)$ & $<0.001^{\dagger}$ & 1 & $1.064(0.693-1.634)$ & 0.777 \\
\hline \multicolumn{7}{|l|}{ Smoking } \\
\hline Never & 1 & $2.066(1.447-2.950)$ & $<0.001^{\dagger}$ & 1 & $0.796(0.500-1.267)$ & 0.335 \\
\hline Former & 1 & $3.106(1.599-6.035)$ & $0.001^{\dagger}$ & 1 & $0.593(0.173-2.032)$ & 0.405 \\
\hline Current & 1 & $2.030(1.125-3.664)$ & 0.019 & 1 & $2.092(0.912-4.798)$ & 0.082 \\
\hline \multicolumn{7}{|c|}{ Alcohol consumption } \\
\hline No & 1 & $2.303(1.686-3.146)$ & $<0.001^{\dagger}$ & 1 & $0.923(0.607-1.404)$ & 0.709 \\
\hline Yes & 1 & $1.933(1.075-3.477)$ & $0.028^{\ddagger}$ & 1 & $1.089(0.458-2.591)$ & 0.848 \\
\hline \multicolumn{7}{|l|}{ Regular exercise } \\
\hline Yes & 1 & $2.508(1.695-3.711)$ & $<0.001^{\dagger}$ & 1 & $0.960(0.572-1.610)$ & 0.877 \\
\hline No & 1 & $1.964(1.336-2.888)$ & $0.001^{\dagger}$ & 1 & $0.986(0.572-1.720)$ & 0.960 \\
\hline \multicolumn{7}{|l|}{ Family income } \\
\hline$<25$ th & 1 & $3.088(1.919-4.971)$ & $<0.001^{\dagger}$ & 1 & $1.192(0.536-2.650)$ & 0.666 \\
\hline 25 th to $<50$ th & 1 & $1.785(1.038-3.068)$ & $0.036^{\ddagger}$ & 1 & $0.833(0.420-1.651)$ & 0.601 \\
\hline 50 th to $<75$ th & 1 & $2.340(1.299-4.215)$ & $0.005^{\dagger}$ & 1 & $1.484(0.626-3.518)$ & 0.370 \\
\hline 75 th to 100 th & 1 & $1.504(0.753-3.001)$ & 0.248 & 1 & $0.780(0.363-1.674)$ & 0.523 \\
\hline
\end{tabular}

Adjusted for age, sex, region, smoking, alcohol consumption, exercise, and family income level. ${ }^{\dagger} P<0.01 .{ }^{\ddagger} P<0.05$.

pants, compared with nonsarcopenic participants, higher HOMA-IR was observed in the 70-79 years age group $(P=0.005)$, higher prevalence of diabetes was shown in the 60-69 and 70-79 years age groups $(P=0.001$ and $P<0.001$, respectively), and MS was more prevalent in the three age groups $(P=0.002, P<0.001$, and $P=0.041$, respectively) (Supplemental Table 1).

\section{Discussion}

Thus far, obesity has been regarded as the most crucial underlying cause of insulin resistance leading to type 2 diabetes and MS. Of note, results of this study demonstrated that sarcopenia may be an early predictor for insulin resistance, diabetes, and MS, even without coexistence of obesity. Sarcopenia showed a significant association with insulin resistance in the nonobese group, but not in the obese group. Sarcopenia was also found to be a risk factor for MS in the nonobese group, but not in the obese-group. In addition, only in the non-obese group, diabetes was more prev- alent among sarcopenic subjects than among those without sarcopenia. All of these findings were applicable particularly to $\geq 60$ years age subgroup. Taken together, our findings indicated that, regarding metabolic disorders, including insulin resistance, diabetes, and MS, non-obese elderly people in the Korean population, compared with obese elderly people, are more affected by sarcopenia. Because people in Asia tend to develop type 2 diabetes with less degree of obesity [30], these facts are worthy of notice.

Although several studies have suggested that insulin resistance and MS are increased synergistically by sarcopenia and obesity [17, 27, 31, 32], the interaction of sarcopenia and obesity in dysglycemia and MS has been inconsistent among previously published studies. One study reported an association of sarcopenia with dysglycemia in obese individuals but not in non-obese individuals [17]. On the contrary, in obese postmenopausal women, sarcopenia showed an association with a better lipid profile and lower CVD predisposing factors [33]. In the New Mexico Aging Process Study, the 
Table 5 Adjusted ORs and 95\% CI of individual MS components by sarcopenia

\begin{tabular}{ccccc}
\hline & Non-obese & $P$ & Obese & $P$ \\
\hline Total participants & & & & \\
Hypertension $^{\mathrm{a}}$ & $1.721(1.279-2.315)$ & $<0.001^{\dagger}$ & $1.332(0.889-1.995)$ & 0.165 \\
Low HDL $^{\mathrm{b}}$ & $1.253(0.957-1.640)$ & 0.101 & $0.746(0.529-1.051)$ & 0.094 \\
Elevated TG $^{\mathrm{c}}$ & $1.550(1.189-2.022)$ & $0.001^{\dagger}$ & $0.771(0.552-1.076)$ & 0.126 \\
Dysglycemia $^{\mathrm{d}}$ & $1.270(0.963-1.675)$ & 0.090 & $1.304(0.929-1.829)$ & 0.124 \\
Central obesity $^{\mathrm{e}}$ & $3.442(2.644-4.481)$ & $<0.001^{\dagger}$ & $2.283(1.077-4.841)$ & $0.031^{\ddagger}$ \\
20-39 years & & & & \\
Hypertension $^{\mathrm{a}}$ & $1.409(0.399-4.971)$ & 0.594 & $2.087(0.832-5.240)$ & 0.117 \\
Low HDL $^{\mathrm{b}}$ & $1.016(0.425-2.427))$ & 0.971 & $0.926(0.361-2.372)$ & 0.872 \\
Elevated TG $^{\mathrm{c}}$ & $1.601(0.613-4.185)$ & 0.337 & $0.842(0.327-2.166)$ & 0.721 \\
Dysglycemia $^{\mathrm{d}}$ & $1.976(0.723-5.403)$ & 0.185 & $1.990(0.808-4.903)$ & 0.135 \\
Central obesity $^{\mathrm{e}}$ & $5.788(2.541-13.187)$ & $<0.001^{\dagger}$ & $1.061(0.296-3.806)$ & 0.928 \\
40-59 years & & & & \\
Hypertension $^{\mathrm{a}}$ & $1.657(1.041-2.637)$ & $0.033^{\ddagger}$ & $1.029(0.578-1.831)$ & 0.922 \\
Low HDL $^{\mathrm{b}}$ & $1.114(0.686-1.809)$ & 0.663 & $0.644(0.357-1.160)$ & 0.143 \\
Elevated TG $^{\mathrm{c}}$ & $1.557(0.977-2.479)$ & 0.062 & $0.746(0.430-1.295)$ & 0.298 \\
Dysglycemia $^{\mathrm{d}}$ & $1.034(0.625-1.709)$ & 0.897 & $1.667(0.961-2.894)$ & 0.069 \\
Central obesity $^{\mathrm{e}}$ & $2.886(1.812-4.596)$ & $<0.001^{\dagger}$ & $2.144(0.810-5.672)$ & 0.125 \\
$\geq 60$ years & & & & \\
Hypertension $^{\mathrm{a}}$ & $2.003(1.313-3.055)$ & $0.001^{\dagger}$ & $1.415(0.677-2.958)$ & 0.356 \\
Low HDL $^{\mathrm{b}}$ & $1.428(0.998-2.043)$ & 0.051 & $0.787(0.480-1.291)$ & 0.343 \\
Elevated TG $^{\mathrm{c}}$ & $1.706(1.210-2.404)$ & $0.002^{\dagger}$ & $0.778(0.482-1.254)$ & 0.303 \\
Dysglycemia $^{\mathrm{d}}$ & $1.451(1.028-2.078)$ & $0.034^{\ddagger}$ & $1.036(0.634-1.694)$ & 0.887 \\
Central obesity $^{\mathrm{e}}$ & $3.862(2.713-5.496)$ & $<0.001^{\dagger}$ & $6.152(0.000-12.757)$ & 0.996 \\
\hline SS $_{\text {metabolic synt }}$ & & & &
\end{tabular}

MS, metabolic syndrome

Adjusted for age, sex, region, smoking, alcohol consumption, exercise, and family income. ${ }^{\text {a} H y p e r t e n s i o n, ~ s y s t o l i c ~} \geq 130 \mathrm{mmHg}$ and/or diastolic $\geq 85 \mathrm{mmHg}$ or on treatment. ${ }^{\mathrm{b}}$ Low $\mathrm{HDL},<40 \mathrm{mg} / \mathrm{dl}$ for men or $<50 \mathrm{mg} / \mathrm{dl}$ for women. ${ }^{\mathrm{c} E l e v a t e d ~ T G . ~} \leq 150 \mathrm{mg} / \mathrm{dL}$ or on treatment. ${ }^{\mathrm{d}}$ Dyglycemia, FPG $\geq 100 \mathrm{mg} / \mathrm{dl}$ or on treatment. ${ }^{\mathrm{e}}$ Central obesity, waist circumference $\geq 90 \mathrm{~cm}$ in men and $\geq 85 \mathrm{~cm}$ in women. ${ }^{\dagger} P<0.01 .{ }^{\dagger} P<0.05$.

prevalence of MS was higher in the non-sacrcopenic obese group than the sarcopenic obese group [34]. The current study showed an additive effect of sarcopenia and obesity on insulin resistance and diabetes, although the effect of sarcopenia on HOMA-IR, MS, and diabetes was insignificant in the obese group. The sarcopenic and obese group showed higher HOMA-IR than either the sarcopenic group or the obese group alone $(3.15 \pm 1.04,2.32 \pm 1.03$, and $3.02 \pm 1.02$, respectively), the prevalence of diabetes in the sarcopenic and obese group was higher than either in the sarcopenic or in the obese group alone $(28.0,23.1$, and $15.2 \%$, respectively). The discrepancy between studies may be, at least in part, due to study design and population characteristics.

Results of the current study showed that non-obese individuals were more affected by sacrcopenia with regard to insulin resistance, and sarcopenia showed a significant association with MS in the non-obese group, but not in the obese group. Diabetes was found to be more prevalent among sarcopenic subjects only in the non-obese group. Obesity is known to be the most important underlying cause of insulin resistance, which is an underlying mechanism for type 2 diabetes and MS. Although sarcopenia may also result in insulin resistance, the effect of sarcopenia is weaker compared with obesity. In the previous studies, obesity without sarcopenia has shown the higher HOMA-IR than sarcopenia without obesity $[17,27]$. In obese subjects, there is greater lipid content within skeletal muscle [35], which may already diminish insulin sensitivity in muscle [36]. Therefore, in that case, sarcopenia may not be a critical determinant for insulin resistance or MS. Therefore we assumed that the pathogenetic mechanism of sarcopenia may differ between in obese individuals and in those without obesity.

No study investigating the association of sarcopenia with insulin resistance or MS specifically in the young age group has been reported, although some studies included subjects who were 20 years of age or older 
in their study population $[17,37]$. One study reported a stronger association of sarcopenia with dysglycemia in individuals younger than 60 years of age, compared with those older than 60 years [17], which is somewhat incompatible with the result of the current study, which did not show a significant association between dysglycemia among metabolic syndrome components with sarcopenia. The age difference in the metabolic effect of sarcopenia is probably due to the result of differences in the etiology of sarcopenia in the young age group, compared with the elderly group. Elderly sarcopenic individuals have more decreased type II muscle fibers, which are less sensitive to insulin action [38, 39], while sarcopenia in younger individuals is caused primarily by reduced accumulation of skeletal muscle mass not by muscle atrophy. Thus, sarcopenia of older individuals could mean increased insulin sensitivity and improved glucose disposal by insulin.

However, in the current study, we did not observe improved insulin resistance in older participants. In contrast, insulin resistance and prevalence of diabetes and MS were higher among sarcopenic individuals than among non-sarcopenic individuals in the elderly group ( $\geq 60$ years). In this study, sarcopenia of older participants, even among non-obese subjects, did not mean increased insulin sensitivity. We did not examine the muscle type of individual participants; therefore, we cannot explain the definite reasons for the discrepancy. We assume that, with regard to metabolic derangements, sarcopenia has the same harmful meaning to elderly subjects as young people. In the current study, the 20-39 years age group showed no significant relationships of sarcopenia with insulin resistance, diabetes, and MS after an adjustment for confounding factors. The prevalence of sarcopenia in the non-obese young age group was very low as $0.009 \%$ in this study, which is inferred as a reason for the insignificant result. The $40-59$ years age group showed significant relationships of sarcopenia with insulin resistance and MS but not with diabetes. It seems that sarcopenia becomes more prevalent and more affects metabolic disorders according to aging. However, it remains to be clarified in the future study.

We adopted ASM divided by body weight (\%) as a skeletal muscle mass index to define sarcopenia, since that ASM/Wt has been widely adopted in many studies to define sarcopenia and that ASM/Wt was suggested to be the more appropriate index for sarcopenic obesity in the Korean population, because ASM/height $(\mathrm{m}) 2$, which was positively correlated with BMI, visceral fat area, and the HOMA-IR, could underestimate sarcopenia in overweight or obese subjects [27].

This study has several limitations. First, we could not differentiate type 1 from type 2 diabetes from the KNHANES dataset. Although we selected participants who were older than 20 years of age and prevalence of type 1 diabetes is relatively low in Korea, we could not exclude the possibility that participants with type 1 diabetes were included in this study. Therefore, there could be selection bias. Second, although we restricted the study population to participants without diagnosed diabetes in order to exclude the effect of diabetes on sarcopenia in certain analyses, the crosssectional study design prevented us from affirming the causal relationship between sarcopenia and insulin resistance, diabetes, and MS. Their causal relationship should be verified in future longitudinal studies. Finally, it was recently suggested that the definition of sarcopenia be restricted to the presence of low muscle strength or poor physical performance in addition to reduced muscle, therefore, because we did not measure muscle strength for determination of sarcopenia; this might also have been a limitation

Despite these limitations, because the population of the current study was representative of the general Korean population, this study has powerful strength. In conclusion, results of this study showed a greater association of sarcopenia with increased insulin resistance and prevalence of diabetes and MS among non-obese participants. Of particular interest, in non-obese elderly people, sarcopenia was found to be a significant correlate of those metabolic disorders. Therefore, sarcopenia may be a significant predictor of type 2 diabetes and MS susceptibility in non-obese elderly population.

\section{Acknowledgement}

This research was supported by a grant from the Daegu and Kyungpook local committee of the Korean Diabetes Association and by the Dongguk University research fund.

\section{Conflicts of Interest}

There are no conflicts of interest.

\section{Disclosure Summary}

The author has nothing to declare. 
Supplemental Table 1 Adjusted geometric means and SEs of HOMA-IR, adjusted ORs and 95\% CIs of prevalence of diabetes and MS according to category of sarcopenia and obesity in elderly subjects

\begin{tabular}{|c|c|c|c|c|c|c|}
\hline & \multicolumn{2}{|c|}{ Non-obese } & \multirow{2}{*}{$P$} & \multicolumn{2}{|c|}{ Obese } & \multirow{2}{*}{$P$} \\
\hline & Non-sarcopenic & Sarcopenic & & Non-sarcopenic & Sarcopenic & \\
\hline \multicolumn{7}{|l|}{ HOMA-IR $^{\mathrm{a}}$} \\
\hline 60-69 year & $2.07 \pm 1.01$ & $2.26 \pm 1.06$ & 0.170 & $3.00 \pm 1.04$ & $3.13 \pm 1.08$ & 0.589 \\
\hline 70-79 year & $2.07 \pm 1.02$ & $2.49 \pm 1.06$ & $0.005^{\dagger}$ & $3.13 \pm 1.07$ & $2.88 \pm 1.10$ & 0.486 \\
\hline$\geq 80$ years & $1.97 \pm 1.04$ & $2.45 \pm 1.13$ & 0.105 & NA & NA & NA \\
\hline \multicolumn{7}{|l|}{ Diabetes } \\
\hline \multicolumn{7}{|l|}{$60-69$ years } \\
\hline Case $/ \mathrm{n}$ & $239 / 1419$ & $21 / 64$ & & $41 / 166$ & $12 / 45$ & \\
\hline Prevalence $(\%)$ & 16.8 & 32.8 & & 24.7 & 26.7 & \\
\hline Adjusted $O R(95 \% \mathrm{CI})^{\mathrm{a}}$ & 1 & $2.528(1.454 \sim 4.398)$ & $0.001^{\dagger}$ & 1 & $0.908(0.403 \sim 2.043)$ & 0.815 \\
\hline \multicolumn{7}{|l|}{$70-79$ years } \\
\hline Case/n & $155 / 852$ & $24 / 65$ & & $30 / 91$ & $23 / 50$ & \\
\hline Prevalence $(\%)$ & 18.2 & 36.9 & & 33.0 & 46.0 & \\
\hline Adjusted $O R(95 \% \mathrm{CI})^{\mathrm{a}}$ & 1 & $2.852(1.641 \sim 4.957)$ & $<0.001^{\dagger}$ & 1 & $1.817(0.854 \sim 3.864)$ & 0.121 \\
\hline \multicolumn{7}{|l|}{$\geq 80$ years } \\
\hline Case/n & $23 / 140$ & $3 / 17$ & & $3 / 7$ & $2 / 5$ & \\
\hline Prevalence $(\%)$ & 16.4 & 5.6 & & 42.9 & 40.0 & \\
\hline Adjusted $O R(95 \% \mathrm{CI})^{\mathrm{a}}$ & 1 & $1.188(0.300 \sim 4.709)$ & 0.806 & 1 & NA & NA \\
\hline \multicolumn{7}{|l|}{ MS } \\
\hline \multicolumn{7}{|l|}{ 60-69 years } \\
\hline Case/n & $516 / 1419$ & $38 / 64$ & & $130 / 166$ & $23 / 45$ & \\
\hline Prevalence $(\%)$ & 36.4 & 59.4 & & 78.3 & 75.6 & \\
\hline Adjusted $O R(95 \% \mathrm{CI})^{\mathrm{a}}$ & 1 & $2.296(1.362 \sim 3.872)$ & $0.002^{\dagger}$ & 1 & $0.867(0.384 \sim 1.957)$ & 0.730 \\
\hline \multicolumn{7}{|l|}{$70-79$ years } \\
\hline Case/n & $320 / 852$ & $42 / 65$ & & $77 / 91$ & $43 / 50$ & \\
\hline Prevalence $(\%)$ & 37.6 & 64.6 & & 84.6 & 86.0 & \\
\hline Adjusted $O R(95 \% \mathrm{CI})^{\mathrm{a}}$ & 1 & $3.041(1.762 \sim 5.246)$ & $<0.001^{\dagger}$ & 1 & $1.035(0.377 \sim 2.841)$ & 0.948 \\
\hline \multicolumn{7}{|l|}{$\geq 80$ years } \\
\hline Case $/ \mathrm{n}$ & $46 / 140$ & $9 / 17$ & & $7 / 7$ & $5 / 5$ & \\
\hline Prevalence $(\%)$ & 32.9 & 52.9 & & 100 & 100 & \\
\hline Adjusted $O R(95 \% \mathrm{CI})^{\mathrm{a}}$ & 1 & $3.183(1.046 \sim 9.684)$ & $0.041^{\dagger}$ & 1 & NA & NA \\
\hline
\end{tabular}

HOMA-IR is presented as adjusted geometric mean $\pm \mathrm{SE}$.

${ }^{a}$ Adjusted for age, sex, region, smoking, alcohol consumption, exercise, and family income. ${ }^{\dagger} P<0.05$. NA, non applicable.

\section{References}

1. Rosenberg IH (1997) Sarcopenia: origins and clinical relevance. J Nutr 127: 990S-991S.

2. Morley JE, Baumgartner RN, Roubenoff R, Mayer J, Nair KS. Sarcopenia. (2001) J Lab Clin Med 137: 231-243.

3. Vanitallie TB (2003) Frailty in the elderly: contributions of sarcopenia and visceral protein depletion. Metabolism 52: $22-26$.

4. Reaven GM (1988) Banting lecture 1988. Role of insulin resistance in human disease. Diabetes 37: 1595-1607.

5. Morley JE (2008) Diabetes, sarcopenia, and frailty. Clin Geriatr Med 24: 455-469.

6. Park SW, Goodpaster BH, Strotmeyer ES, de Rekeneire N, Harris TB, et al. (2006) Decreased muscle strength and quality in older adults with type 2 diabetes: the health, aging, and body composition study. Diabetes 55: 18131818.

7. Park SW, Goodpaster BH, Lee JS, Kuller LH, Boudreau R, et al. (2009) Excessive loss of skeletal muscle mass in older adults with type 2 diabetes. Diabetes Care 32: 1993-1997.

8. Lee CG, Boyko EJ, Strotmeyer ES, Lewis CE, Cawthon PM, et al. (2011) Association between insulin resistance and lean mass loss and fat mass gain in older men without diabetes mellitus. J Am Geriatr Soc 59: 1217-1224.

9. Klip A, Paquet MR (1990) Glucose transport and glucose transporters in muscle and their metabolic regulation. Diabetes Care 13: 228-243.

10. Walsh K (2009) Adipokines, myokines and cardiovascular disease. Circ J 73: 13-18.

11. Pedersen BK (2010) Muscle-to-fat interaction: a two-way street? J Physiol 588: 21.

12. Solerte SB, Gazzaruso C, Bonacasa R, Rondanelli M, Zamboni M, et al. (2008) Nutritional supplements with oral amino acid mixtures increases whole-body lean mass and insulin sensitivity in elderly subjects with sarcopenia. Am J Cardiol 101: 69E-77E. 
13. Cornier MA, Dabelea D, Hernandez TL, Lindstrom RC, Steig AJ, et al. (2008) The metabolic syndrome. Endocr Rev 29: 777-822.

14. Gallagher D, Visser M, De Meersman RE, Sepulveda D, Baumgartner RN, et al. (1997) Appendicular skeletal muscle mass: effects of age, gender, and ethnicity. J Appl Physiol 83: 229-239.

15. Lexell J, Henriksson-Larsen K, Winblad B, Sjostrom M (1983) Distribution of different fiber types in human skeletal muscles: effects of aging studied in whole muscle cross sections. Muscle Nerve 6: 588-595.

16. Klitgaard H, Zhou M, Schiaffino S, Betto R, Salviati G, et al. (1990) Ageing alters the myosin heavy chain composition of single fibres from human skeletal muscle. Acta Physiol Scand 140: 55-62.

17. Srikanthan P, Hevener AL, Karlamangla AS (2010) Sarcopenia exacerbates obesity-associated insulin resistance and dysglycemia: findings from the National Health and Nutrition Examination Survey III. PLoS One 5: e10805.

18. Yoon YS, Oh SW, Baik HW, Park HS, Kim WY (2004) Alcohol consumption and the metabolic syndrome in Korean adults: the 1998 Korean National Health and Nutrition Examination Survey. Am J Clin Nutr 80: 217224.

19. Choi HS, Oh HJ, Choi H, Choi WH, Kim JG, et al. (2010) Vitamin D insufficiency in Korea--a greater threat to younger generation: the Korea National Health and Nutrition Examination Survey (KNHANES) 2008. J Clin Endocrinol Metab 96: 643-651.

20. Kim Y, Lee BK (2012) Associations of blood lead, cadmium, and mercury with estimated glomerular filtration rate in the Korean general population: Analysis of 20082010 Korean National Health and Nutrition Examination Survey data. Environ Res 118: 124-129.

21. Matthews DR, Hosker JP, Rudenski AS, Naylor BA, Treacher DF, et al. (1985) Homeostasis model assessment: insulin resistance and beta-cell function from fasting plasma glucose and insulin concentrations in man. Diabetologia 28: 412-419.

22. Zheng W, McLerran DF, Rolland B, Zhang X, Inoue M, et al. (2011) Association between body-mass index and risk of death in more than 1 million Asians. $N$ Engl J Med 364: 719-729.

23. (2004) Appropriate body-mass index for Asian populations and its implications for policy and intervention strategies. Lancet 363: 157-163.

24. Grundy SM, Cleeman JI, Daniels SR, Donato KA, Eckel RH, et al. (2005) Diagnosis and management of the metabolic syndrome: an American Heart Association/National Heart, Lung, and Blood Institute Scientific Statement. Circulation 112: 2735-2752.

25. Lee SY, Park HS, Kim DJ, Han JH, Kim SM, et al. (2007) Appropriate waist circumference cutoff points for central obesity in Korean adults. Diabetes Res Clin Pract 75:
$72-80$.

26. Pietrobelli A, Formica C, Wang Z, Heymsfield SB (1996) Dual-energy X-ray absorptiometry body composition model: review of physical concepts. Am J Physiol 271: E941-951.

27. Lim S, Kim JH, Yoon JW, Kang SM, Choi SH, et al. (2010) Sarcopenic obesity: prevalence and association with metabolic syndrome in the Korean Longitudinal Study on Health and Aging (KLoSHA). Diabetes Care 33: 1652-1654.

28. Muscaritoli M, Anker SD, Argiles J, Aversa Z, Bauer JM, et al. (2010) Consensus definition of sarcopenia, cachexia and pre-cachexia: joint document elaborated by Special Interest Groups (SIG) "cachexia-anorexia in chronic wasting diseases" and "nutrition in geriatrics". Clin Nutr 29: 154-159.

29. Janssen I, Heymsfield SB, Ross R (2002) Low relative skeletal muscle mass (sarcopenia) in older persons is associated with functional impairment and physical disability. J Am Geriatr Soc 50: 889-896.

30. Yoon KH, Lee JH, Kim JW, Cho JH, Choi YH, et al. (2006) Epidemic obesity and type 2 diabetes in Asia. Lancet 368: 1681-1688.

31. Zamboni M, Mazzali G, Fantin F, Rossi A, Di Francesco V (2008) Sarcopenic obesity: a new category of obesity in the elderly. Nutr Metab Cardiovasc Dis 18: 388-395.

32. Stenholm S, Harris TB, Rantanen T, Visser M, Kritchevsky SB, et al. (2008) Sarcopenic obesity: definition, cause and consequences. Curr Opin Clin Nutr Metab Care 11: 693700 .

33. Aubertin-Leheudre M, Lord C, Goulet ED, Khalil A, Dionne IJ (2006) Effect of sarcopenia on cardiovascular disease risk factors in obese postmenopausal women. Obesity (Silver Spring) 14: 2277-2283.

34. Baumgartner RN, Wayne SJ, Waters DL, Janssen I, Gallagher D, et al (2004) Sarcopenic obesity predicts instrumental activities of daily living disability in the elderly. Obes Res 12: 1995-2004.

35. Goodpaster BH, Theriault R, Watkins SC, Kelley DE (2000) Intramuscular lipid content is increased in obesity and decreased by weight loss. Metabolism 49: 467-472.

36. Petersen KF, Befroy D, Dufour S, Dziura J, Ariyan C, et al.(2003) Mitochondrial dysfunction in the elderly: possible role in insulin resistance. Science 300: 1140-1142.

37. Kim TN, Park MS, Lim KI, Choi HY, Yang SJ, et al. (2013) Relationships between Sarcopenic Obesity and Insulin Resistance, Inflammation, and Vitamin D Status:The Korean Sarcopenic Obesity Study (KSOS). Clin Endocrinol (Oxf) 78: 525-532.

38. Nader GA, Esser KA (2001) Intracellular signaling specificity in skeletal muscle in response to different modes of exercise. J Appl Physiol 90: 1936-1942.

39. Narici MV, Maffulli N (2010) Sarcopenia: characteristics, mechanisms and functional significance. Br Med Bull 95: 139-159. 